\title{
Pore distribution and classification of loess
}

\author{
Qianqian Liu ${ }^{1}$, and Tiehang Wang ${ }^{1}$ \\ ${ }^{1}$ School of Civil Engineering, Xi 'an University of Architecture and Technology, Xi 'an, Shaanxi, China
}

\begin{abstract}
The micro-pore characteristics in undisturbed loess have a profound influence on the permeability characteristics. Based on the results of permeability test, it is proved that the permeability coefficient of soil samples with the same porosity ratio is different and the internal characteristics are diversified. The internal pores of undisturbed loess were studied by scanning electron microscopy (SEM). The results show that there are obvious differences in the internal microstructure of the original loess with the same porosity ratio. There are root holes in undisturbed loess, the number and size of which directly affect the value of permeability coefficient. With the increase of pore diameter, the pore size distribution changes gradually, and the influence of small pores is gradually weakened, while the influence of large pores is more obvious. Therefore, only the pore ratio parameter used in engineering can not completely describe the pore characteristics of soil, it is necessary to introduce other microscopic parameters to describe.
\end{abstract}

\section{Introduction}

Loess is a kind of porous medium with obvious permeability. The pore size distribution (PSD) of loess is affected by the initial moisture content, soil structure, mineral composition and stress history ${ }^{[1-2]}$. As a seepage channel, the pore size, connectivity and the proportion of pore volume in the soil will affect the permeability of soil, that is, the permeability of soil mainly depends on the size of soil pores and the microscopic characteristics of pores. Permeability test results show that the permeability results of undisturbed loess with the same porosity ratio are different, and the permeability coefficient of undisturbed loess with root holes is obviously greater than that of soil samples with uniform pores. Based on this result, the internal pore characteristics of undisturbed loess are studied.

So far, there are many experimental methods to obtain the microscopic characteristics of materials, such as scanning electron microscopy (SEM), mercury injection method, adsorption method, X-ray diffraction method, isothermal adsorption method, nuclear magnetic resonance method, light microscopy, etc ${ }^{[3-4]}$. Scanning electron microscopy (SEM) is one of the most effe1ctive methods to clearly observe the image of pores, and the general distribution of pores in soil can be observed by SEM. In this chapter, scanning electron microscopy (SEM) is used to study the microscopic pore distribution characteristics of unaltered loess with different pore ratios and different directions, and to analyze the differences of pore characteristics of soils with the same pore ratios and the same direction.

\section{Test soil sample scheme and prepara- tion}

The unaltered loess from a foundation pit project site in $\mathrm{Xi}$ 'an was used in this test. The basic information of the soil sample was as follows: Q3 loess-like soil, light yellow, plastic and wet, with different densities within the depth range and plasticity index ranging from 11.7 to 12.3. Due to the little difference in plasticity index, the soil in the sampling depth range can be considered to have basically the same particle composition. The basic physical parameters of loess were obtained through tests. All test methods strictly abide by the Standard of Geotechnical Test Methods (GB/T50123-2019). Its basic physical parameters are shown in Table 1, the test scheme is shown in Table 2, and the test results of particle analysis are shown in Figure 1.

Table 1. Main physical properties of loess

\begin{tabular}{|c|c|c|c|}
\hline$G_{S}$ & 2.71 & $I_{p}$ & $11.7 \sim 12.3$ \\
\hline$W_{L} / \%$ & 29.8 & $w$ & $9 \% \sim 16 \%$ \\
\hline$W_{P} / \%$ & 17.7 & $\mathrm{e}$ & $0.7 \sim 1.1$ \\
\hline
\end{tabular}

*Liu Qianqian (1996-), female, master candidate, mainly engaged in the research of foundation.

E-mail: 1509255833@qq.com 


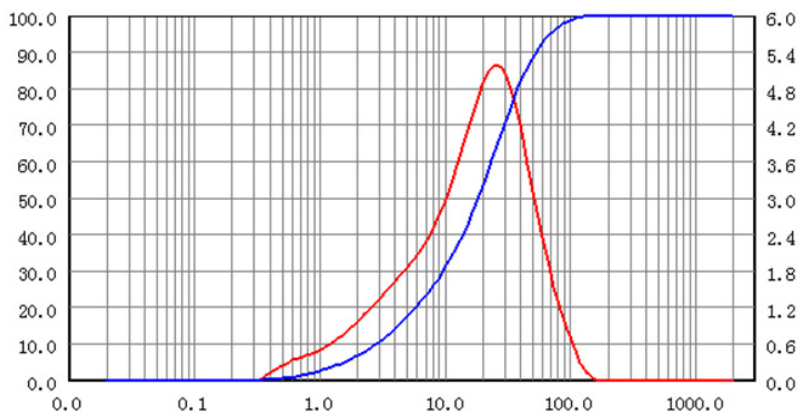

Fig. 1 Experimental results of loess particle analysis

Table 2 Sample number and porosity ratio

\begin{tabular}{|c|c|c|c|c|}
\hline $\begin{array}{c}\text { Serial } \\
\text { number }\end{array}$ & A1 & A2 & A3 & A4 \\
\hline$e$ & 0.84 & 0.91 & 1.03 & 1.16 \\
\hline
\end{tabular}

\section{Scanning electron microscope test results and analysis}

In this experiment, 3 ring samples per pore ratio were prepared and taken from 3 different soils, and 4 scanning samples were taken from the ring samples, 2 of which were taken from the same ring samples. Among them, scanning samples A4-1 and A4-2 are taken from the same ring cutter, while scanning samples A4-3 and A4-4 are taken from different ring cutters. The porosity of scanning samples is obtained through image processing software ImagePro-Plus. The experimental results show that the porosity of the scanning samples taken from the same ring knife is basically the same, and the relative error of controlling the porosity is less than $10 \%$ by data analysis, and the test repeatability is good. The porosity of scanned samples taken from different rings varies. Although the pore ratio of these four samples is not significantly different, the internal differences are quite obvious.

Table 3 Porosity after image processing

\begin{tabular}{|c|c|c|c|c|}
\hline $\begin{array}{c}\text { Serial } \\
\text { number }\end{array}$ & A4-1 & A4-2 & A4-3 & A4-4 \\
\hline $\begin{array}{c}\text { Surface } \\
\text { porosity }\end{array}$ & 0.25 & 0.25 & 0.21 & 0.18 \\
\hline
\end{tabular}

According to the pore characteristics analysis results of the above soil samples, the curve shown in Fig. 2 is drawn, in which the pore density is the percentage content of the area within the diameter of a certain hole. As can be seen from Fig. 2 (a), the pore diameter distribution range of the soil sample is concentrated in the range of $100 \sim 10000 \mu \mathrm{m}$ and presents a kurtosis distribution. Figure 2 (b) shows the relationship between the number of pores and the porosity ratio. It can be seen from the figure that there is not much relationship between the number of pores and the porosity ratio. It can be seen from the figures (a) and (b) that the internal structural characteristics of soil samples with the same porosity ratio are diversified. The sample A1, A2 and A3 all showed similar regularity. Fig. 2 (c) $\sim$ (e).

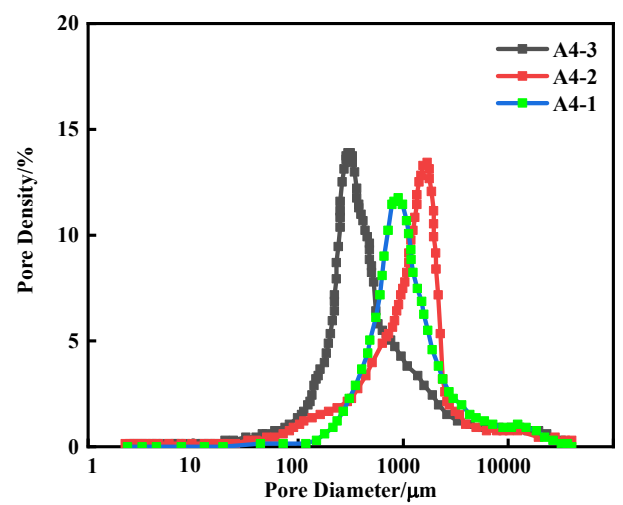

(a) Pore density distribution curve of sample A4

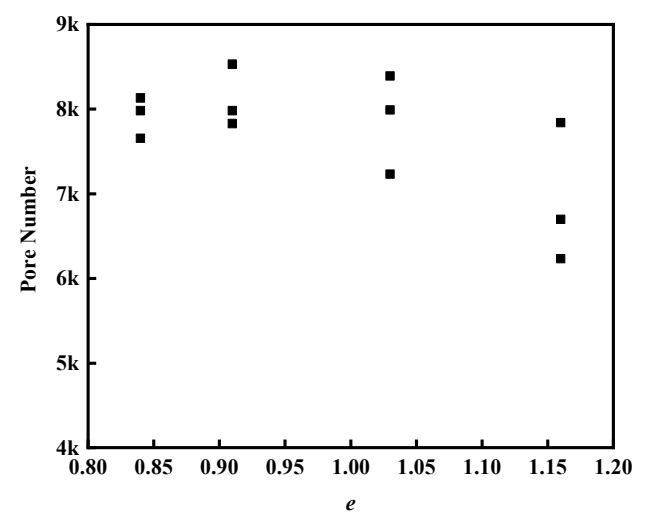

(b) Pore quantity distribution diagram of the sample 


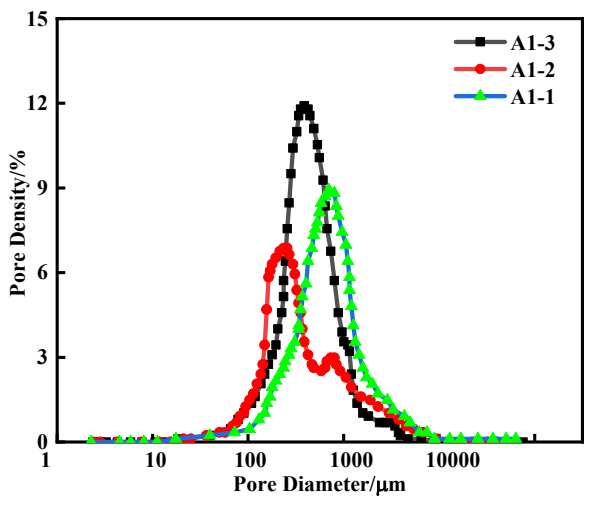

(c) Pore density distribution curve of sample A1

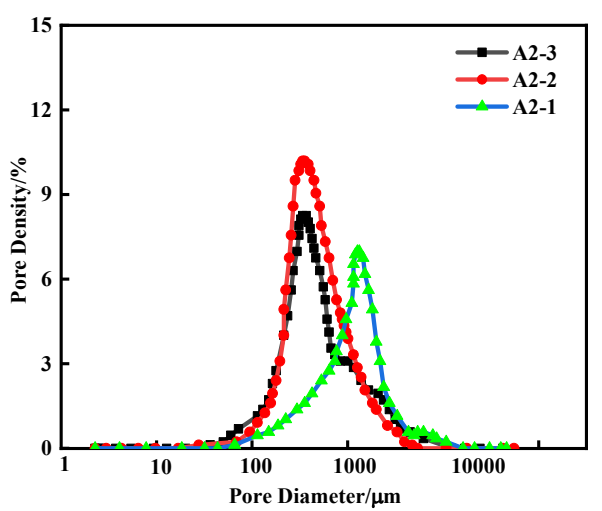

(d) Pore density distribution curve of sample A2

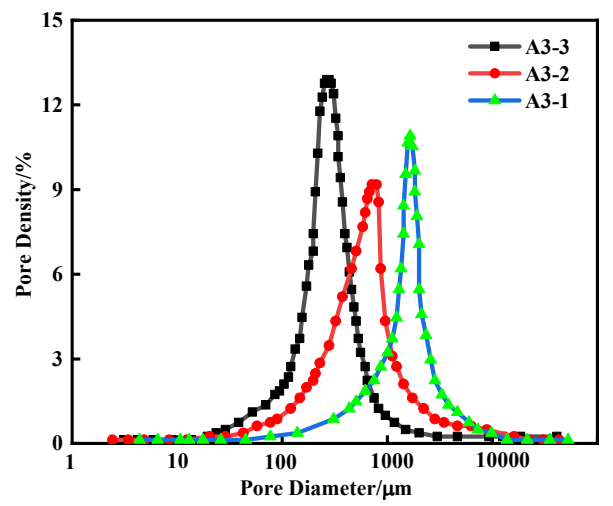

(e) Pore density distribution curve of sample A3

Fig. 2 Results of pore distribution characteristics

\subsection{Relationship between pore characteristics and pore surface area}

In order to more clearly see the specific situation of the pore distribution of the soil sample, Figure 2 (a) (d) respectively show the physical images of the pore distribution of the unintact soil sample with different pore ratios, from which we can clearly see the general situation of the pore distribution.

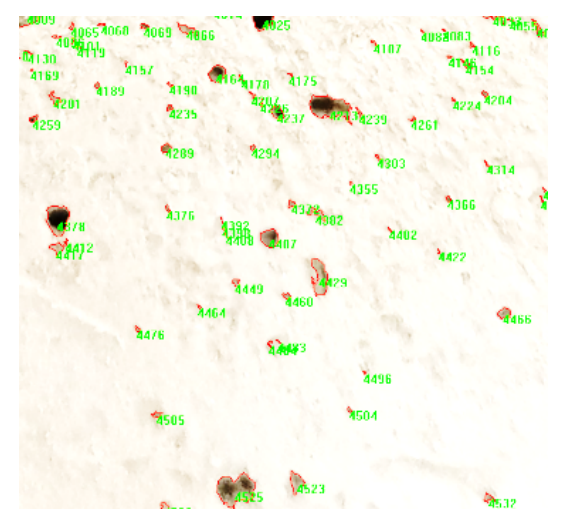

(a)

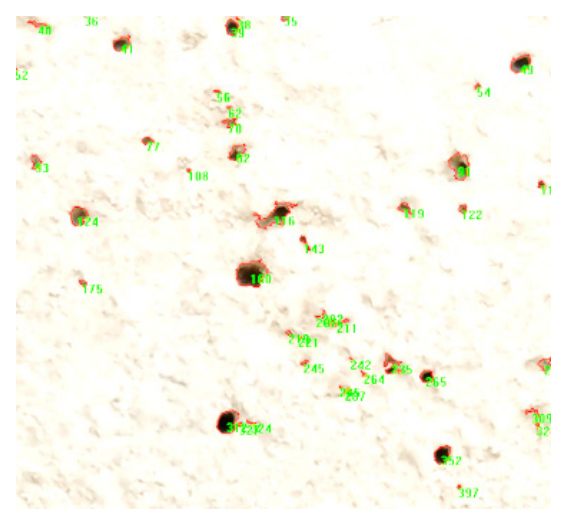

(b)

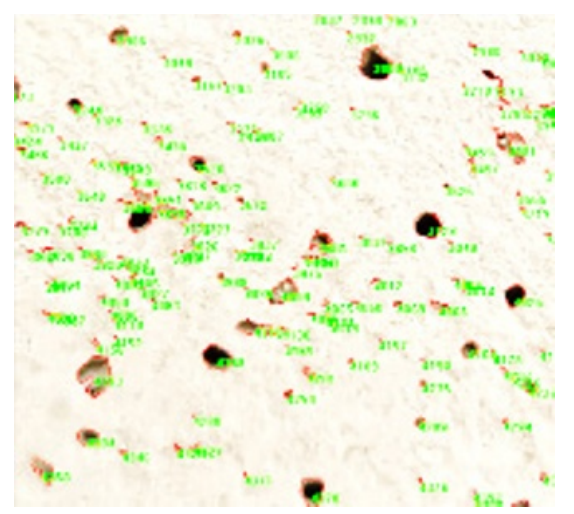

(c)

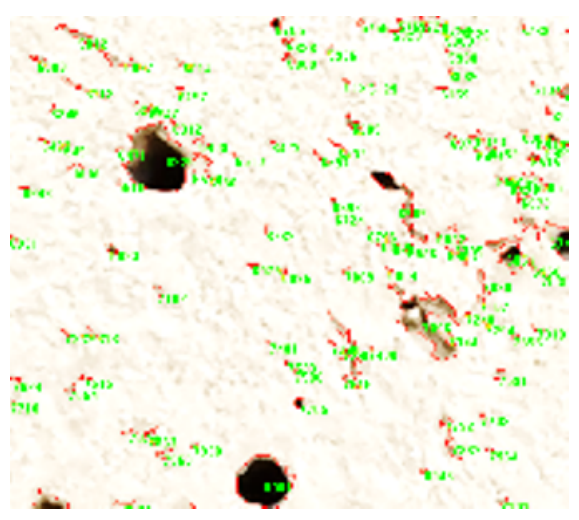

(d)

Fig. 3 Local picture of undisturbed loess sample

Image processing software Matlab and ImageProPlus are used for image preprocessing, image noise reduction, 
image segmentation and other work. the characteristics of pore distribution histogram as shown in figure 4.

As shown in the figure, based on the treatment results of each sample, it is found that the aperture peak value of the three samples of $\mathrm{A} 1$ is around $500 \mu \mathrm{m}, 1600 \mu \mathrm{m}$ and $2300 \mu \mathrm{m}$. As can be seen from the figure, the pore distribution of soil samples corresponding to the peak value of $500 \mu \mathrm{m}$ is relatively uniform, and no pores can be clearly recognized by naked eyes. The soil samples corresponding to the peak value of $1600 \mu \mathrm{m}$ have visible pores with small pore size, which is obviously different from the soil samples corresponding to the peak value of $600 \mu \mathrm{m}$. Peak of 2300 microns with visible pores in soil samples, there is a pore $>=2000$ microns in diameter, can through the light of the ground, it is a has a root hole (through hole) of soil samples, and the other two soil samples are significantly different, the soil samples in less than 2000 microns of porosity, pore area is mainly caused by the root hole, which may account for the similar pore ratio of undisturbed loess internal differences, these differences will lead to differences of other property. The pore diameters of A2, A3 and A4 samples also showed similar distribution patterns. The pore diameters of (a) were all before the range of $1000 \mu \mathrm{m}$, (b) were all before the range of $2000 \mu \mathrm{m}$, and (c) were all before the range of $3000 \mu \mathrm{m}$.

The same void ratio test of test result shows that the diversity of the internal pore characteristics of loess, separately USES the expression in the macro parameters of soil pore void ratio is not a complete description of the porosity of the soil characteristics, the need to introduce such as pore size and distribution, pore distribution and connectivity, twists, and other microscopic parameters can be better to describe the characteristics of the soil, it is also the future research microscopic characteristics and permeability of soil and soil consolidation compression deformation features such as the important direction.

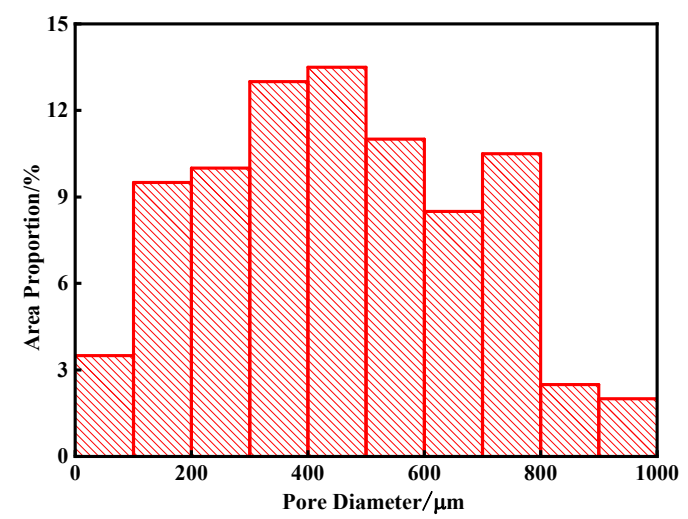

(a)

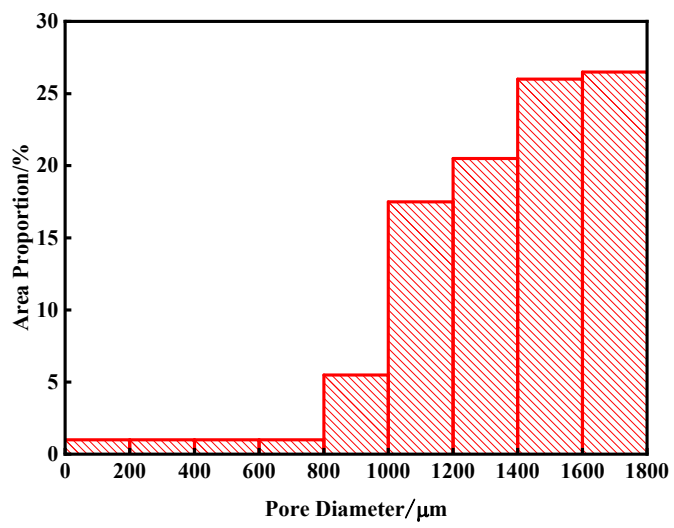

(b)

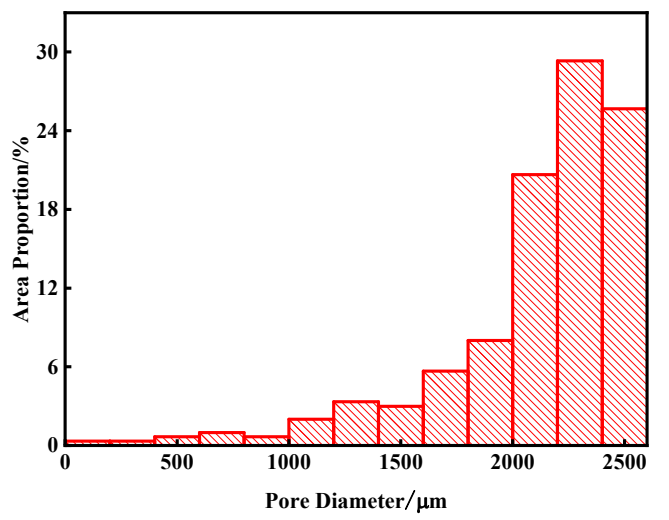

(c)

Fig. 4 Histogram of pore distribution characteristics

\subsection{Relationship between pore characteristics and root pore}

In this chapter, according to the pore size range corresponding to the pore area percentage, the pores are classified as follows by referring to relevant literature ${ }^{[5]}$ : (1) small pores (diameter $<1000 \mu \mathrm{m}$ ) are mainly intergranular pores, (2) medium pores $(1000 \mu \mathrm{m} \leq \mathrm{D}<$ $2000 \mu \mathrm{m}$ ) are mainly fine root pores of plants, (3) large pores $(\mathrm{D} \geq 2000 \mu \mathrm{m})$ are mainly coarse root pores of plants.

According to the above classification criteria for soil, the hundred components of various kinds of pores in different soil samples will be obtained, as shown in Table 2-3. Among them, the area percentage of pore diameter corresponds to more than $60 \%$ of the division, the range of the first kind of the diameter of the pore are mainly concentrated in the range of less than 1000 microns, the second category of the diameter of the pore are mainly concentrated in the range of $1000 \sim 2000$ microns, the third type of the diameter of the pore are mainly concentrated in the scope of more than 2000 microns, the void ratio under the same conditions, with the emergence of large porosity, small pore content reduce, small pore relative weakening the influence of the nature of soil, soil macropore appearance has changed the structure characteristic and some important 
properties, such as infiltration characteristics, strength characteristics and so on. Coarse root holes are through holes in the soil, have straight, oblique and curved, the third type of porosity corresponds to the root of the size of hole, the first type of porosity corresponds to the minimum volume, the second times, pore size and the internal structure characteristics of the soil has obvious effects on its permeability, especially the appearance of the thick root hole permeability increase greatly, therefore contains coarse root hole soil has good drainage function. The above test results show that the pore characteristics are diverse, and it is necessary to carefully analyze the influence of these pores on the foundation in the engineering, and use different microscopic parameters to describe the conclusion to be more accurate, which plays a practical guiding role in foundation treatment.

Table 4. Formatting sections, subsections and subsubsections

\begin{tabular}{|c|c|c|c|c|c|c|c|c|c|c|c|c|}
\hline \multirow{2}{*}{ Percent } & \multicolumn{3}{|c|}{ A1 } & \multicolumn{3}{|c|}{$\mathrm{A} 2$} & \multicolumn{3}{|c|}{ A3 } & \multicolumn{3}{|c|}{ A4 } \\
\hline & A1-1 & A1-2 & A3-1 & A3-2 & A3-3 & A4-1 & A3-1 & A3-2 & A3-3 & A4-1 & A4-2 & A4-3 \\
\hline $\begin{array}{l}\text { Large } \\
\text { Pores }\end{array}$ & 0 & 0 & 0 & 0 & 74.79 & 0 & 0 & 0 & 74.79 & 0 & 0 & 86.95 \\
\hline $\begin{array}{l}\text { Medium } \\
\text { Pores }\end{array}$ & 0 & 91.28 & 0 & 78.52 & 21.99 & 0 & 0 & 78.52 & 21.99 & 0 & 75.23 & 9.86 \\
\hline $\begin{array}{l}\text { Small } \\
\text { Pores }\end{array}$ & 100 & 8.72 & 100 & 21.48 & 3.22 & 100 & 100 & 21.48 & 3.22 & 100 & 24.77 & 3.19 \\
\hline
\end{tabular}

\subsection{Relationship between pore characteristics and root pore}

Void ratio of the soil is one of the important parameters of pore characteristics of soil samples, but as a result of scanning electron microscopy (sem) to calculate the pore ratio and three-phase pore ratio error to a certain extent, there are a lot of literature analyses error ${ }^{[6]}$, if no error, scanning electron microscopy (sem) in theory the resulting void ratio should be slightly less than three phase calculation of void ratio. According to the relevant literature, the author summarizes the reasons for the errors as follows:

(1) Error of physical and mechanical parameters of soil samples. The tests for specific gravity and water content contain errors, which lead to the error between the three-phase calculation of the porosity ratio and the real porosity ratio.

(2) Error introduced in the sample preparation process of ring knife soil sample. The prepared ring knife soil sample cannot guarantee that each is absolutely consistent, and the same ring knife soil sample cannot guarantee that the interior is completely uniform and consistent.

(3) Limitations of scanning electron microscopy experiment. The theoretical assumption of the cylindrical pore model is different from the actual one. What is measured is the volume content corresponding to the pore diameter. The range of pore diameter that can be measured by scanning electron microscopy determines the effectiveness of this experiment for the measurement of macropores, and it is not possible to measure the pore diameter smaller than $1 \mu \mathrm{m}$ by scanning electron microscopy.

To sum up, errors are unavoidable. In this paper, the data analyzed are used to control the relative error of the porosity ratio within $10 \%$. In addition, normalization is adopted for the analysis and comparison of data.

\section{Conclusion}

In this chapter, scanning electron microscopy (SEM) was used to study the microscopic pore structure characteristics of loess soil particles with four kinds of pore ratios under the condition of the same pore ratio, and the influence of the thickness of root pores on the pore characteristics of soil was analyzed. The following conclusions were drawn:

(1) The distribution function of the number of pores shows the type of unimodal pores. With the increase of the pore ratio, the pore diameter corresponding to the peak increases, and the proportion of macropores gradually increases.

(2) According to the pore characteristics and pore distribution rules of the soil samples, the pores of the undisturbed loess studied in this chapter are divided into three levels: large pores, medium pores and small pores.

(3) The pore characteristics of loess with the same porosity ratio are diverse. Pore ratio alone cannot describe the internal differences of soil, and other parameters, such as pore diameter distribution range, pore connectivity, and humpness, need to be introduced for better description.

\section{References}

1. Zhang H.Urban Construction Theory Research (Electronic Edition), 34,187-197(2018)

2. Kong JP, Hu HJ, Fan HH. Journal of Seismological Engineering, 38,903-908. (2016)

3. Yan XQ, Fang YG, Zhang P. E.Chinese Journal of Geotechnical Engineering, 33,1302-1307(2011)

4. Zhang XZ, Lu T, Lu YD, Lu YC, Li X, Pan WS. Bulletin of Soil and Water Conservation,38,212-216 (2018)

5. Sun Y, Zhao DF. Unconventional Oil and Gas, 7,914(2020) 
6. Li TL, Zhang H, Li P, Kang HW, Ge SL. Hydrogeology and Engineering Geology,47,107114(2020) 